\title{
Calculation of Surface Plasmon Effect on Optical Discs
}

\author{
Kuo Pin ChiU*, Wei Chih Lin, Yuan Hsing Fu and Din Ping TsAi
}

Center of Nanostorage Research, National Taiwan University, Taipei 10617, Taiwan

Department of Physics, National Taiwan University, Taipei 10617, Taiwan

(Received December 8, 2003; revised January 9, 2004; accepted January 23, 2004; published July 29, 2004)

We calculated the conditions of surface plasmon resonance for various optical discs to evaluate the role of periodic lands and grooves or recording mark trains as grating couplers. With the typical parameters of structure used in the discs, it is shown that surface plasmons have a weak influence on ordinary optical discs. These results differ from the situation with super-resolution near-field optical structures where surface plasmons or localized surface plasmons have a considerable influence on such discs. [DOI: 10.1143/JJAP.43.4730]

KEYWORDS: surface plasmon, optical discs

\section{Introduction}

The interaction of electromagnetic radiation with an interface can generate several interesting phenomena. In 1902, Wood reported the observation of a series of anomalies in spectra from ruled metallic diffraction gratings. ${ }^{1)}$ One of these anomalies took the form of sharp changes in the reflected intensity of transverse magnetic (TM) polarized radiation when the grating vector was parallel to the plane of incidence. It is now well established that the "anomalous" spectra arise from the incident electromagnetic wave coupling to the surface plasmons (SPs) excited at the interface. SPs are electromagnetic excitations that propagate along the interface between a metal and a dielectric. Recently, SPs have been found to play an important role in the trapping of incident radiation into small volumes and enhancing the transmitted light through a thin film. ${ }^{2)}$ In 2001, Tominaga et al. mentioned the possibility of the coupling of SPs by the recorded mark trains in $\mathrm{AgO}_{x}$-type super-resolution near-field optical structures (super-RENS). ${ }^{3}$ The effect of surface plasmon resonance further enhances signal intensity. On the other hand, nanoscale noble-metal structures can exhibit anomalous optical excitation in the visible range due to a localized surface plasmon (LSP). ${ }^{4-8)}$ The LSP resonance positions depend mainly on the structural geometry and polarization state of incident light. LSPs were suggested to be the key factor in super-RENS. ${ }^{9)}$ In this study, we investigated SPs and LSPs that may result from the various features of compact disc (CD) and digital versatile disc (DVD). The structure of optical discs, such as periodic lands and grooves, and the recorded marks may serve as a grating coupler for an incident laser to excite SPs by providing the in-plane momentum needed to excite of SPs. The effects of these SPs may affect the readout signals in the far field and near field.

\section{Basic Principle of Calculation}

A surface plasmon is an electromagnetic excitation at the interface between a material with negative permittivity (often a metal) and a dielectric. It consists of a surface charge density oscillation coupled to electromagnetic fields which are bound to the surface and decay exponentially into both media. Consider a flat interface that is formed by combining a dielectric and a metal with permittivities $\varepsilon_{1}$ and

*E-mail address: kpchiu@ntu.edu.tw $\varepsilon_{2}$ respectively. It is possible to calculate the dispersion relations of the surface plasmon using Maxwell's equation of electromagnetic waves together with the boundary conditions of $\mathbf{E}$ and $\mathbf{H}$ fields at the interface. ${ }^{10)}$

$$
k_{x}=\frac{\omega}{c}\left(\frac{\varepsilon_{1} \varepsilon_{2}}{\varepsilon_{1}+\varepsilon_{2}}\right)^{1 / 2} \equiv k_{\mathrm{sp}}
$$

and

$$
k_{z i}=\left[\varepsilon_{i}\left(\frac{\omega}{c}\right)^{2}-k_{x}^{2}\right] ; i=1,2 .
$$

In eq. (1), $k_{x}$ is the wave vector of the surface plasmon along the direction parallel to the interface. $k_{z 1}$ and $k_{z 2}$ are the wave vectors of the surface plasmon in the direction perpendicular to the interface in the dielectric (with permittivity $\varepsilon_{1}$ ) and in the metal (with permittivity $\varepsilon_{2}$ ), respectively. $\omega$ is the frequency of incident light, and $c$ is the velocity of light in a vacuum. Equation (1) shows that the $k_{x}$ of the surface plasma exceeds that of incident light having the same frequency within the adjacent dielectric, as shown in Fig. 1(a). This result means that a surface plasmon on a single flat interface between a metal and a dielectric cannot be excited simply by radiating light onto that surface. To excite surface plasmons it is necessary to have an appropriate coupling geometry. For a periodically corrugated dielectric/metal interface with period $a$, as shown in Fig. 1(b), the wave vector of the incident light may effectively gain the integer values of the grating vector, $g$, of periodic corrugations. The relation can be expressed as

$$
k_{x}=\frac{\omega}{c} \sqrt{\varepsilon_{1}} \sin \theta+n g,
$$

with

$$
g=\frac{2 \pi}{a} \quad \text { and } \quad n=1,2,3, \ldots
$$

where $\theta$ is the incident angle of impinging light. When the $k_{x}$ of impinging light differs from that of the surface plasmons by a nonzero value of the grating vector, incident light may couple to the surface plasmons.

\section{Structures and Parameters of Optical Discs}

Ordinary optical discs have multilayer structures containing protective layers, a recording layer, a reflective layer and a polycarbonate (PC) substrate. Figure 2 shows a schematic diagram of ordinary optical discs. The reflective layer is usually made of $\mathrm{Al}$ alloy, and each protective layer is made 


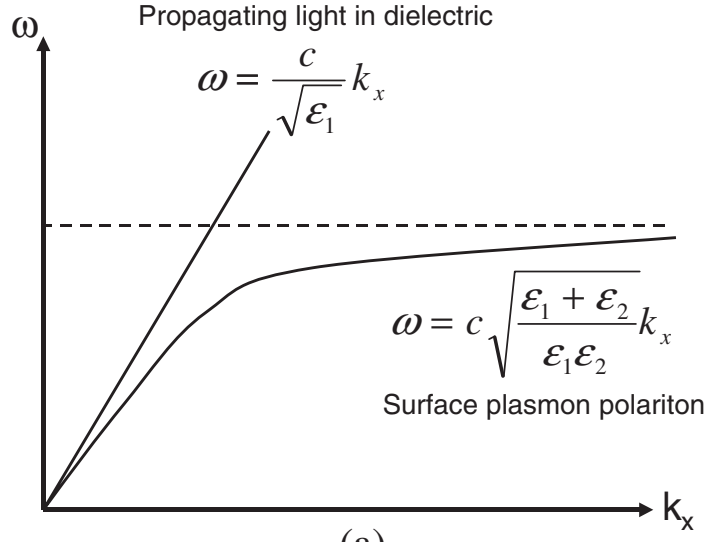

(a)

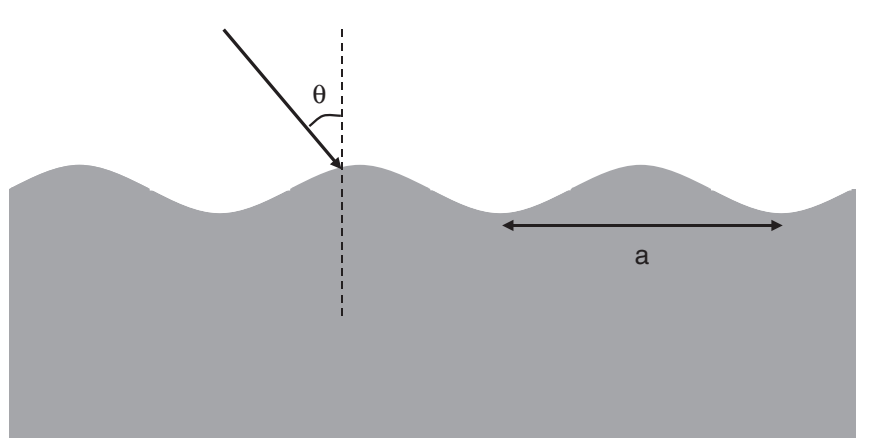

(b)

Fig. 1. (a) Dispersion curve of surface plasmons. (b) Excitation of surface plasmons by a grating coupler. The grating period is $a$, and the incident angle of impinging light is $\theta$. A periodic structure can provide the in-plane momentum required for incident light of appropriate polarization to excite a surface plasmon.

of $\mathrm{ZnS}-\mathrm{SiO}_{2}$ composites. The recording layers of DVD recordable (DVD-R) and phase change discs are made of a dye and a phase-change alloy, respectively. The periodic land-groove structure of these discs serves as a grating coupler, which may provide the in-plane momentum needed to excite surface plasmons along the radial direction of the discs. On the other hand, the recording mark trains in the recording layer may also serve as the grating coupler in the tangential direction of the discs. In the following calculations, the dielectric constants at different wavelengths of each layered material are cited from previous reports. ${ }^{11-17)}$

\section{Results and Discussion}

We have calculated the surface plasmon resonance conditions satisfied by various types of disc structure. All the calculations are based on the simple consideration that the interface is within two infinite media. Table I shows our calculation results in the radial direction of the optical discs. The first column is the type of disc being considered. The following $\lambda$ and numerical aperture (NA) values are the corresponding laser wavelengths and numerical apertures that are typically used in each disc. Because the periodic land-groove structure exists in layers shown in Fig. 2 for the different types of disc, we consider each interface separately in Table I. The quantity $k_{x, \max }$ is the maximum $k_{x}$ of incident light, as seen in Fig. 3(a). It is calculated using

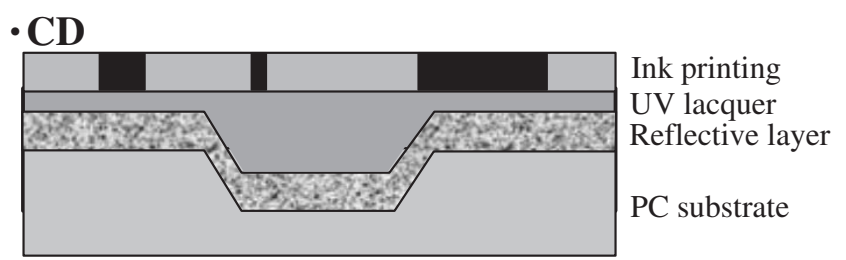

(a)

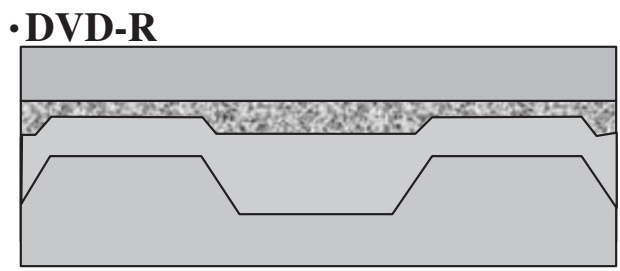

Protection layer Reflective layer Dye

(b)

\section{-Phase change disc}

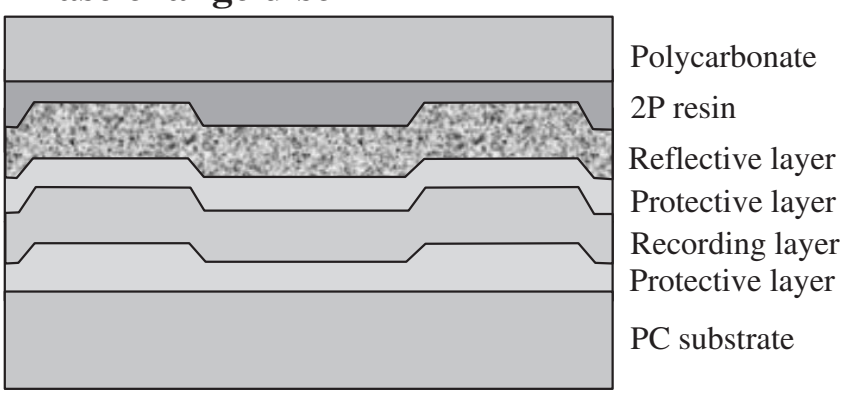

(c)

Fig. 2. Schematic diagram of the structure of optical discs: (a) CD, (b) DVD-R and (c) phase-change discs, such as CD-RW, DVD-RAM, DVDRW and DVD+RW.

$$
k_{x, \max }=\frac{2 \pi}{\lambda} \times \mathrm{NA} .
$$

With the incident angle of the focused laser beam ranging from zero to the maximum angle, $\theta_{\mathrm{NA}}, k_{x}$ ranges from zero to $k_{x, \text { max }}$ for a given NA. Because the dielectric constants adopted are complex numbers, ${ }^{11-17)}$ the $k_{\mathrm{sp}}, k_{\mathrm{z} 1}$ and $k_{\mathrm{z} 2}$ calculated from eq. (1) are also complex. The real part of these quantities is denoted by an additional "-r" in the lower index, and the imaginary part is denoted by an additional "$i$ " in the lower index as can be seen in the first row of Table I. The real parts of $k_{\mathrm{sp}}$ and $k_{\mathrm{z}}$ represent the propagating properties of surface plasmon waves, and the imaginary parts of $k_{\mathrm{sp}}$ and $k_{\mathrm{z}}$ are related to the dissipation of electromagnetic energy.

The $a_{\max }$ in the table is the maximum period in the landgroove structure, i.e., the maximum track pitch, which allows incident light to excite the surface plasmons at the corresponding interface. It is calculated using

$$
k_{\mathrm{sp}-\mathrm{r}}=k_{x, \max }+\frac{2 \pi}{a_{\max }},
$$

where $k_{\text {sp-r }}$ and $k_{x, \max }$ are those calculated previously. This relation comes from eq. (2) by adopting $n=1$; that is, we consider the first-order diffraction of incident light only. Because the $k_{x}$ of incident light with the given NA is smaller than $k_{x, \max }$, the track pitch must be smaller than $a_{\max }$ to allow 
Table I. Calculation results of surface plasmon resonance conditions for optical discs in the radial direction.

\begin{tabular}{|c|c|c|c|c|c|c|c|c|c|c|c|c|c|}
\hline Disk & $\begin{array}{c}\lambda \\
(\mathrm{nm})\end{array}$ & NA & $\begin{array}{l}\text { Track pitch } \\
\qquad(\mu \mathrm{m})\end{array}$ & interface & $\begin{array}{c}k_{\mathrm{x}, \max } \\
\left(\times 10^{6} \mathrm{~m}^{-1}\right)\end{array}$ & $\begin{array}{c}k_{\mathrm{sp}-\mathrm{r}} \\
\left(\times 10^{6} \mathrm{~m}^{-1}\right)\end{array}$ & $\begin{array}{c}k_{\mathrm{sp}-\mathrm{i}} \\
\left(\times 10^{6} \mathrm{~m}^{-1}\right)\end{array}$ & $\begin{array}{c}k_{\mathrm{z} 1-\mathrm{r}} \\
\left(\times 10^{6} \mathrm{~m}^{-1}\right)\end{array}$ & $\begin{array}{c}k_{\mathrm{zl}-\mathrm{i}} \\
\left(\times 10^{6} \mathrm{~m}^{-1}\right)\end{array}$ & $\begin{array}{c}k_{\mathrm{z2} 2 \mathrm{-r}} \\
\left(\times 10^{6} \mathrm{~m}^{-1}\right)\end{array}$ & $\begin{array}{c}k_{\mathrm{z2} 2 \mathrm{i}} \\
\left(\times 10^{6} \mathrm{~m}^{-1}\right)\end{array}$ & $\begin{array}{l}a_{\max } \\
(\mu \mathrm{m})\end{array}$ & $\begin{array}{c}\sin \theta_{\min } \\
(a=\text { track pitch })\end{array}$ \\
\hline CD-ROM & 780 & 0.45 & 1.6 & $\mathrm{Al} / \mathrm{PC}$ & 3.623 & 12.886 & 0.155 & 15.035 & 58.346 & 0.752 & -2.652 & 0.678 & 1.113 \\
\hline \multirow{3}{*}{ DVR-R } & \multirow{3}{*}{635} & \multirow{3}{*}{0.6} & \multirow{3}{*}{0.74} & $\mathrm{ZnS}-\mathrm{SiO}_{2} / \mathrm{Al}$ & 5.934 & 22.510 & 0.480 & 1.482 & -7.289 & 10.720 & 67.311 & 0.379 & 1.418 \\
\hline & & & & $\mathrm{Al} /$ Dye & 5.934 & $\ldots$ & $\ldots$ & $\ldots$ & $\ldots$ & $\ldots$ & $\ldots$ & $\ldots$ & $\ldots$ \\
\hline & & & & Dye/PC & 5.934 & $\ldots$ & $\ldots$ & $\ldots$ & $\ldots$ & $\ldots$ & $\ldots$ & $\ldots$ & $\ldots$ \\
\hline \multirow{4}{*}{ CD-RW } & \multirow{4}{*}{780} & \multirow{4}{*}{0.45} & \multirow{4}{*}{1.6} & $\mathrm{PC} / \mathrm{Al}$ & 3.623 & 12.886 & 0.155 & 0.752 & -2.652 & 15.035 & 58.346 & 0.678 & 1.113 \\
\hline & & & & $\mathrm{Al} / \mathrm{ZnS}-\mathrm{SiO}_{2}$ & 3.623 & 17.743 & 0.404 & 14.654 & 59.511 & 1.455 & -4.929 & 0.445 & 1.716 \\
\hline & & & & $\mathrm{ZnS}-\mathrm{SiO}_{2} / \mathrm{GST}$ & 3.623 & 16.782 & 0.718 & 4.357 & -2.766 & 43.486 & 33.140 & 0.477 & 1.597 \\
\hline & & & & $\mathrm{ZnS}-\mathrm{SiO}_{2} / \mathrm{PC}$ & 3.623 & 10.161 & 0.000 & 13.756 & 0.000 & 7.505 & 0.000 & 0.961 & 0.774 \\
\hline \multirow{8}{*}{ DVD-RW } & \multirow{4}{*}{650} & \multirow{4}{*}{0.6} & \multirow{4}{*}{0.74} & $\mathrm{PC} / \mathrm{Al}$ & 5.796 & 15.629 & 0.165 & 0.716 & -3.596 & 11.640 & 6.561 & 0.639 & 0.739 \\
\hline & & & & $\mathrm{Al} / \mathrm{ZnS}-\mathrm{SiO}_{2}$ & 5.796 & 21.861 & 0.451 & 11.239 & 67.294 & 1.439 & -6.858 & 0.391 & 1.384 \\
\hline & & & & 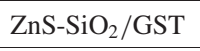 & 5.796 & 20.558 & 1.109 & 5.399 & -4.222 & 44.333 & 43.115 & 0.426 & 1.249 \\
\hline & & & & $\mathrm{ZnS}-\mathrm{SiO}_{2} / \mathrm{PC}$ & 5.796 & 12.286 & 0.000 & 16.786 & 0.000 & 8.993 & 0.000 & 0.968 & 0.393 \\
\hline & \multirow{4}{*}{635} & \multirow{4}{*}{0.6} & \multirow{4}{*}{0.74} & $\mathrm{PC} / \mathrm{Al}$ & 5.934 & 16.025 & 0.173 & 0.730 & -3.796 & 11.139 & 65.500 & 0.623 & 0.762 \\
\hline & & & & $\mathrm{Al} / \mathrm{ZnS}-\mathrm{SiO}_{2}$ & 5.934 & 22.510 & 0.480 & 10.720 & 67.311 & 1.482 & -7.289 & 0.379 & 1.418 \\
\hline & & & & 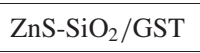 & 5.934 & 26.176 & 2.284 & 8.884 & -6.730 & 42.155 & 45.393 & 0.310 & 1.788 \\
\hline & & & & $\mathrm{ZnS}-\mathrm{SiO}_{2} / \mathrm{PC}$ & 5.934 & 13.462 & 0.000 & 23.074 & 0.000 & 7.854 & 0.000 & 0.835 & 0.503 \\
\hline \multirow{4}{*}{ DVD-RAM } & \multirow{4}{*}{650} & \multirow{4}{*}{0.6} & \multirow{4}{*}{0.615} & $\mathrm{PC} / \mathrm{Al}$ & 5.796 & 15.629 & 0.165 & 0.716 & -3.596 & 11.640 & 6.561 & 0.639 & 0.560 \\
\hline & & & & $\mathrm{Al} / \mathrm{ZnS}-\mathrm{SiO}_{2}$ & 5.796 & 21.861 & 0.451 & 11.239 & 67.294 & 1.439 & -6.858 & 0.391 & 1.205 \\
\hline & & & & $\mathrm{ZnS}-\mathrm{SiO}_{2} / \mathrm{GST}$ & 5.796 & 20.558 & 1.109 & 5.399 & -4.222 & 44.333 & 43.115 & 0.426 & 1.071 \\
\hline & & & & $\mathrm{ZnS}-\mathrm{SiO}_{2} / \mathrm{PC}$ & 5.796 & 12.286 & 0.000 & 16.786 & 0.000 & 8.993 & 0.000 & 0.968 & 0.214 \\
\hline
\end{tabular}




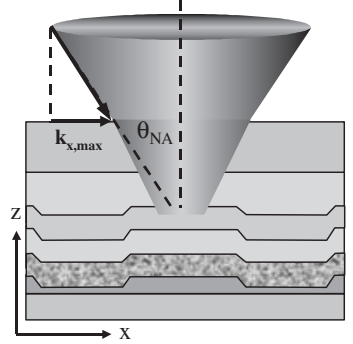

(a)

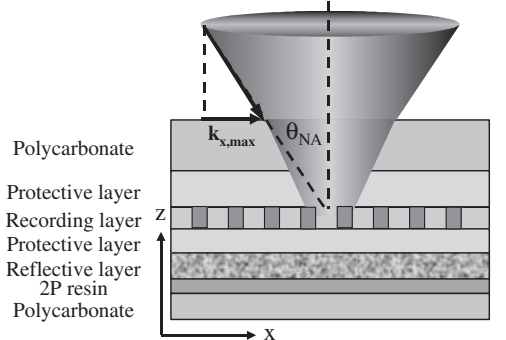

(b)
Fig. 3. Schematic diagram of an incident laser beam (wavelength $\lambda$ ) focused by an objective (NA, $1 \times \sin \theta_{\mathrm{NA}}$ ) on an optical disc. (a) The $x$ direction is along the radial direction of the disc. (b) The $x$-direction is along the tangential direction of the disc.

the laser beam to excite surface plasmons at the interface. On the other hand, if the period of the land-groove structure is set to the typical value of track pitch, the incident angle must be greater than some minimum value for the laser beam to excite surface plasmons of the interface. This minimum angle can be calculated using

$$
k_{\mathrm{sp}-\mathrm{r}}=\frac{2 \pi}{\lambda} \times \sin \theta_{\min }+\frac{2 \pi}{\text { track pitch }},
$$

where the values of $k_{\mathrm{sp}-\mathrm{r}}, \lambda$ and track pitch are given in the table. Similar to the calculation of $a_{\max }$, in eq. (5), we consider only the first-order diffraction of incident light. It should be noted that, when the laser beam is focused by the objective in air, the $\sin \theta_{\min }$ obtained using eq. (5) is the minimum NA needed to excite surface plasmons at the corresponding interface.

From the results of $a_{\max }$ in Table I, it can be seen that for typically used parameters of some optical discs, such as CD read-only-memory (CD-ROM), DVD-R and CD rewritable (CD-RW), there will be no surface plasmon effect at every interface. However, it is possible to excite surface plasmons at the $\mathrm{ZnS}-\mathrm{SiO}_{2} / \mathrm{PC}$ interface in DVD rewritable (DVD$\mathrm{RW}$ ) and at the $\mathrm{ZnS}-\mathrm{SiO}_{2} / \mathrm{PC}$ and $\mathrm{PC} / \mathrm{Al}$ interfaces in DVD random-access-memory (DVD-RAM) because the track pitch in these instances is smaller than $a_{\max }$. When considering the values of $\sin \theta_{\min }$ in Table I, we find that, by changing NA, it is possible to excite surface plasmons at some additional interfaces other than the interfaces described above. If we can increase the NA of CD-RW to 0.774 , surface plasmons may be excited at the $\mathrm{ZnS}-\mathrm{SiO}_{2} / \mathrm{PC}$ interface of the disc. Results show that the NA is increased to 0.739 for a laser wavelength of $650 \mathrm{~nm}$, or to 0.762 for a laser wavelength of $635 \mathrm{~nm}$, and that surface plasmons can be excited at the $\mathrm{PC} / \mathrm{Al}$ interface of the DVD-RW disc as well.

We also calculated the surface plasmon resonance conditions in the tangential direction of various optical discs. The calculation results are shown in Table II. Because there is no periodic land-groove structure in the tangential direction of the disc, the recording mark trains can serve as grating couplers. Because the recording mark trains are located in the PC layer of CD and in the phase-change alloy recording layer, such as in GeSbTe of DVD-RW, we consider only the $\mathrm{Al} / \mathrm{PC}$ interface for $\mathrm{CD}$ and $\mathrm{ZnS}-\mathrm{SiO}_{2} /$ GeSbTe interface for phase change discs, as shown in

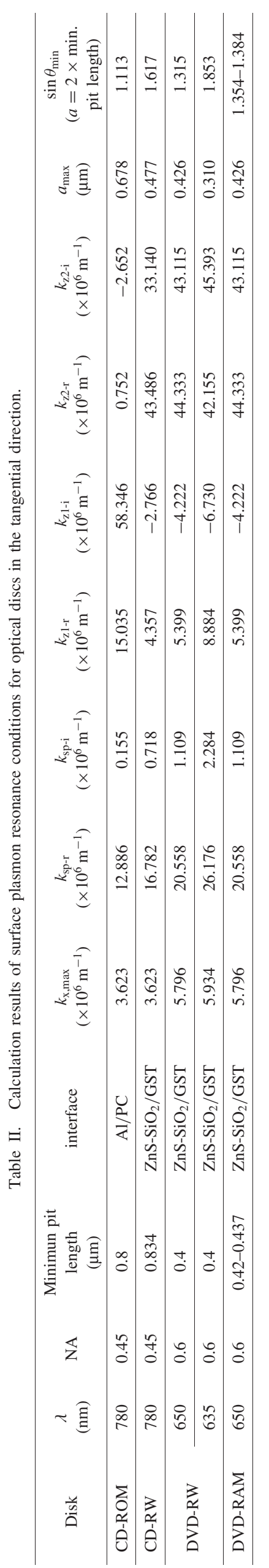




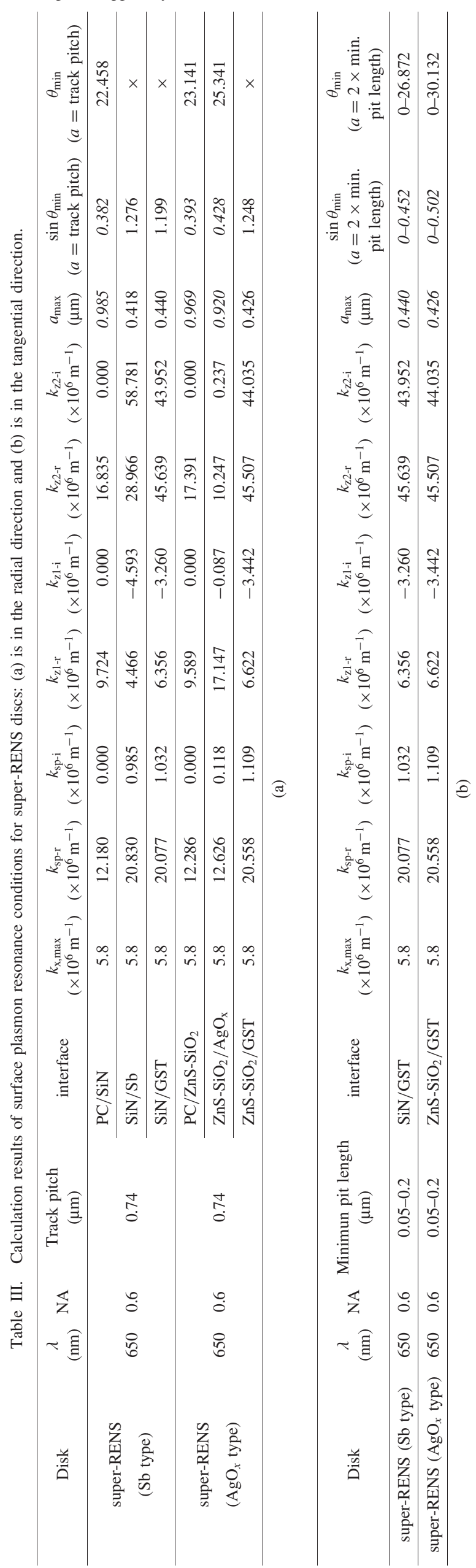

Table II. In this case, we view the minimum pit length of the recording marks as one-half the period of the grating structure.

The calculations of $k_{x, \max }, k_{\mathrm{sp}}, k_{\mathrm{z} 1}, k_{\mathrm{z} 2}$ and $a_{\max }$ in Table II use the same relations as the calculations in the case of the radial direction. For the calculation of $\sin \theta_{\min }$, we also use the eq. (5), but replace the track pitch with double the minimum pit length. As in the discussion on surface plasmon resonance condition in the radial direction, surface plasmons can be excited at the corresponding interface when the minimum pit length is smaller than one-half the $a_{\max }$. As the results show in Table II, it can be seen that there is no surface plasmon effect on the interfaces of the ordinary optical discs in the tangential direction as well.

For the purpose of comparison, we also calculate the surface plasmon resonance conditions for two types of super-RENS discs using antimony ( $\mathrm{Sb}$ ) and silver oxide $\left(\mathrm{AgO}_{x}\right)$ as the active layers. Table III(a) shows the results in the radial direction of the super-RENS discs. We can see that there are surface plasma modes at the $\mathrm{PC} / \mathrm{SiN}$ interface of $\mathrm{Sb}$-type super-RENS and at $\mathrm{PC} / \mathrm{ZnS}-\mathrm{SiO}_{2}$ and $\mathrm{ZnS}-\mathrm{SiO}_{2} /$ $\mathrm{AgO}_{x}$ interfaces of $\mathrm{AgO}_{x}$-type super-RENS. For the tangential direction, the results are shown in Table III(b). Because the minimum pit length of the recording mark train in superRENS disc can be very small (ranging from $50 \mathrm{~nm}$ to $200 \mathrm{~nm}$ ), the surface plasmons will be excited in both types of super-RENS disc.

Because the surface plasmon can propagate along the interface, it will dissipate the energy density of the focusing spot, and it may also increase the crosstalk between signals. ${ }^{18-21)}$ The carrier-to-noise ratio (CNR) of the disc can be seriously affected by the interactions of surface plasmons. The absence of the interactions of surface plasmons should be one of the guidelines for commercial discs. However, for the recently developed ultrahigh-density optical disc such as the super-RENS, ${ }^{3,5-9)}$ the working mechanism of near-field optical recording results from the influence of localized surface plasmons. The signal intensities of nanoscale marks are considered to be enhanced by localized surface plasmons. The details of the thickness of layered materials, the size and shape of local structures and the localized interactions of the random distribution have a considerable influence on the generation of the localized surface plasmons..$^{5-9)}$ The read and write properties of optical signals are strongly related to localized surface plasmons in the case of the super-RENS. The destruction and construction of the interactions of surface plasmons are different for various types of disc structure. The advantages and disadvantages of the surface plasmon and localized surface plasmon effects are mixed and very structuredependent.

\section{Conclusions}

We have calculated the wave vectors $\left(k_{\mathrm{sp}}\right)$ of surface plasmon at each interface of different optical discs. The maximum period $\left(a_{\max }\right)$ or minimum NA $\left(\sin \theta_{\min }\right)$ required to excite surface plasmons on several discs has been calculated. For the generally used parameters, only DVDRAM has surface plasmon modes in the radial direction of the disc at the PC/Al interface. There are some modes of surface waves in the radial directions of DVD-RW and 
DVD-RAM as well. Because the surface plasmons may decrease the CNR of disc, they are not found in the formats and parameters used by commercial discs, as we expected. A super-resolution optical disc with a nanometer scale track pitch and pit length may experience the surface plasmon effect, and localized surface plasmons are definitely needed for nanooptical interactions and the surmounting of surface plasmons.

\section{Acknowledgement}

We thank the National Science Council, Taiwan, R.O.C. for financial support under grant number NSC-92-2120-M002-008, and the Ministry of Economic Affairs of Taiwan, R.O.C. under grant number 92-EC-17-A-08-S1-0006.

1) R. W. Wood: Philos. Mag. 4 (1902) 396.

2) F. J. García-Vidal and Martín-Moreno: Phys. Rev. B 66 (2002) 155412.

3) J. Tominaga, J. Kim, H. Fuji, D. Büchel, T. Kikukawa, L. Men, H. Fukuda, A. Sato, T. Nakano, A. Tachibana, Y. Yamakawa, M. Kumagai, T. Fukaya and N. Atoda: Jpn. J. Appl. Phys. 40 (2001) 1831.

4) D. P. Tsai, J. Kovacs, Z. Wang, M. Moskovits, V. M. Shalaev, J. S. Suh and R. Botet: Phys. Rev. Lett. 72 (1994) 4149.
5) D. P. Tsai, C. W. Yang, W. C. Lin, F. H. Ho, H. J. Huang, M. Y. Chen, T. F. Tseng, C. H. Lee and C. J. Yeh: Jpn. J. Appl. Phys. 39 (2000) 982.

6) W. C. Liu, C. Y. Wen, K. H. Chen, W. C. Lin and D. P. Tsai: Appl. Phys. Lett. 78 (2001) 685.

7) T. Fukaya, D. Buechel, S. Shinbori, J. Tominaga, N. Atoda, D. P. Tsai and W. C. Lin: J. Appl. Phys. 89 (2001) 6139.

8) W. C. Liu and D. P. Tsai: Jpn. J. Appl. Phys. 42 (2003) 1031.

9) W. C. Liu and D. P. Tsai: Phys. Rev. B 65 (2002) 155423.

10) H. Rather: Surface Plasmons on Smooth and Rough Surfaces and Gratings (Springer-Verlag, Berlin, 1998).

11) R. J. Martín-Palma and J. M. Martínez-Duart: J. Vac. Sci. \& Technol. A 16 (1998) 409.

12) N. Yamada, E. Ohno, K. Nishiuchi and N. Akahira: J. Appl. Phys. 69 (1991) 2849.

13) C. Peng, L. Cheng and M. Mansuripur: J. Appl. Phys. 82 (1997) 4183.

14) E. García-García: J. Vac. Sci. \& Technol. A 17 (1999) 1805.

15) D. V. Tsu: J. Vac. Sci. \& Technol. A 17 (1999) 1854.

16) A. Mendoza-Galván and J. González-Hernández: J. Appl. Phys. 87 (2000) 760 .

17) P. K. Khulbe, E. M. Wright and M. Mansuripur: J. Appl. Phys. 88 (2000) 3926.

18) R. E. Gerber, L. Li and M. Mansuripur: Appl. Opt. 34 (1995) 4929.

19) W. C. Liu and M. W. Kowarz: Appl. Opt. 38 (1999) 3787.

20) J. M. Brok and H. P. Urbach: J. Opt. Soc. Am. A 20 (2003) 256.

21) E. Fontana: Appl. Opt. 43 (2004) 79. 